\title{
Reduced mobility of di-propylene glycol methylether in its aqueous mixtures by quasielastic neutron scattering
}

\author{
Jan Swenson, ${ }^{1}$ Johan Sjöström, ${ }^{1}$ and Felix Fernandez-Alonso ${ }^{2}$ \\ ${ }^{1}$ Department of Applied Physics, Chalmers University of Technology, SE-41296 Göteborg, Sweden \\ ${ }^{2}$ ISIS Facility, Rutherford Appleton Laboratory, Chilton, Didcot, OX11 0QX Oxfordshire, United Kingdom
}

(Received 8 July 2010; accepted 24 October 2010; published online 20 December 2010)

\begin{abstract}
The hydrogen (H-) bonding interplay between water and other organic molecules is important both in nature and in a wide range of technological applications. Structural relaxation and, thus, diffusion in aqueous mixtures are generally dependent on both the strength and the structure of the H-bonds. To investigate diffusion in $\mathrm{H}$-bonding mixtures, we present a quasielastic neutron scattering study of di-propylene glycol methylether (2PGME) mixed with $\mathrm{H}_{2} \mathrm{O}$ (or $\mathrm{D}_{2} \mathrm{O}$ ) over the concentration range 0-90 wt.\% water. We observe a nonmonotonic behavior of the dynamics with a maximum in average relaxation time for the mixture with $30 \mathrm{wt}$. $\%$ water, which is more than a factor 2 larger compared to that of either of the pure constituents. This is a result in qualitative agreement with previous calorimetric studies and the behavior of aqueous mixtures of simple mono-alcohols. More surprisingly, we notice that the dynamics of the 2PGME molecules in the mixture is slowed down by more than a factor 3 at $30 \mathrm{wt} . \%$ water but that the water dynamics indicates an almost monotonous behavior. Furthermore, in the low momentum transfer $(Q)$ range of the 2PGME, where the intermediate scattering function $I(Q, t)$ is considerably stretched in time (i.e., the stretching parameter $\beta \ll 1$ ), it is evident for the $2 \mathrm{PGME}-\mathrm{D}_{2} \mathrm{O}$ samples that the $Q$-dependence of the inverse average relaxation time, $\langle\tau\rangle^{-1}$, is greater than 2 . This implies that the relaxation dynamics is partly homogenously stretched, i.e., the relaxation of each relaxing unit is somewhat intrinsically stretched in time. () 2010 American Institute of Physics. [doi:10.1063/1.3515958]
\end{abstract}

\section{INTRODUCTION}

Life, as we know it, would have not been possible without the many anomalous properties of water. Furthermore, since it is the hydrogen bonds between the water molecules that are responsible for many peculiar properties of water, this means that it is the unique properties of the hydrogen bonds that make life possible. In fact, if the strength of this bond, which is about $0.24 \mathrm{eV},{ }^{1}$ had been considerably higher or lower, we would not have existed. Thus, the importance of hydrogen bonds for life, nature, and technology cannot be understated. ${ }^{2}$

A common approach to explore the role of the hydrogen bond and its specific properties is to study binary liquids, where one or both components display hydrogen bonding. In this way, it is possible to perform systematic variations of the hydrogen-bond density and to investigate how such alterations can affect the structural and dynamical properties of the liquid. Common model systems are alcoholwater mixtures, where the viscosity of the mixture exhibits an anomalous concentration dependence with a maximum in viscosity at typically about 25 wt.\% water $^{3-5}$ and substantial negative excess entropy compared to ideal mixing. ${ }^{6,7}$ The unusual behavior has been explained by the hydrophobic interaction between alcohol molecules and surrounding water molecules, ${ }^{8-11}$ which leads to both slower water dynamics $^{12-14}$ and a higher local average density ${ }^{8-11}$ of the two molecular water layers closest to the alcohol molecules. In fact, the concentration dependence of the viscosity displays the same behavior as the deviation from a linear density dependence. ${ }^{15}$ Also, other mixtures such as water-dimethyl sulfoxide (DMSO) (Ref. 16) have shown a similar viscosity maximum at intermediate concentrations. However, for most of these systems, including alcohol-water mixtures, it appears as if it is mainly the reduced diffusivity of the water dynamics (both translational and rotational motions) that is responsible for the viscosity maximum. The dynamics of the other component shows a more linear concentration dependence. ${ }^{16}$

Another useful model system, which has been used previously for systematic studies of the role of hydrogen bonds, consists of aqueous mixtures of $n$-propylene glycol methylether ( $n$ PGME), $n=1,2,3$, where the density of hydrogen bonds can be varied by both the chain-length, $n$, and the concentration of $n$ PGME in solution. ${ }^{17,18}$ As for the mono-alcohols, each $n$ PGME molecule possesses one hydroxyl group with the possibility to donate one and accept two H-bonds. In pure $n$ PGME, the molecules appear to dynamically pair up forming effective relaxing units of two H-bonded molecules. This is indicated by comparing, e.g., structural relaxations of the oligomeric $n$ PGME series to those of $n$-propylene dimethyl ethers ( $n \mathrm{PGDE}$ ), which are the same systems without the ability to form hydrogen bonds. ${ }^{19,20}$

For low concentrations of water, we expect the water molecules to reside where they can form H-bonds. Thus, the water molecules are likely to form $\mathrm{H}$-bond to the single $\mathrm{OH}$ end-group of an $n$ PGME molecule. Hence, a water cluster, with an average size depending on the water concentration, is likely to act as a bridge between $n$ PGME molecules, and thereby form relatively larger effective units that relax as single units. ${ }^{18}$ The $n$ PGME molecules thus bond mostly to the 


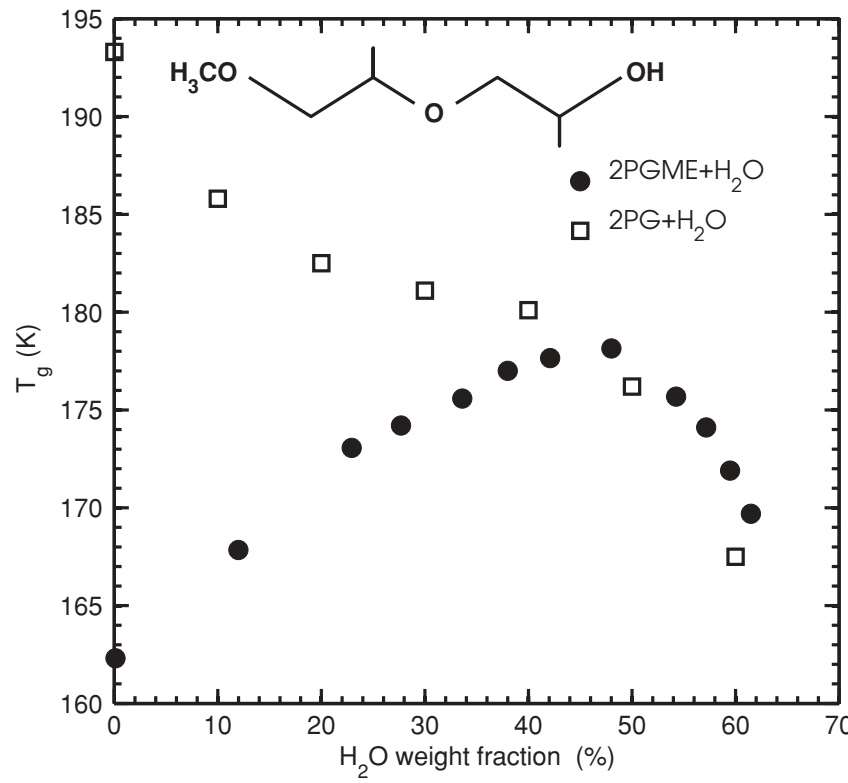

FIG. 1. Glass transition temperatures, $T_{g}$, vs the weight fraction of water in the 2PGME-water and 2PG-water systems. A clear maximum in $T_{g}$ is observed at about $45 \mathrm{wt} . \%$ water for the $2 \mathrm{PGME}$ system. The chemical structure of 2 PGME is also shown.

bridging cluster of water molecules and not to each other. In support for the existence of such clusters is the concentration dependence of the glass transition temperature, $T_{g}$, in these systems, which shows a similar behavior as the viscosity of the alcohol-water mixtures discussed above. Figure 1 shows how $T_{g}$ changes with the weight fraction of water in the 2PGME-water system. ${ }^{18}$ The dramatic increase of $T_{g}$ by $16 \mathrm{~K}$ up to the $T_{g}$ maximum at about $45 \mathrm{wt} . \%$ water can be compared with a more normal behavior for aqueous dipropylene glycol (2PG, with two OH end-groups), where infinitely large chains or clusters of hydrogen bonded molecules can be formed at all concentrations. The substantial increase of $T_{g}$ in the 2PGME-water system reflects an increase in size of the hydrogen-bonded clusters described above.

The existence of similar structures has been shown for water-methanol mixtures. Detailed neutron diffraction studies, interpreted with the empirical potential structure refinement scheme, ${ }^{21}$ show that instead of methanol $\mathrm{OH}$ groups bonding and forming chains as in the pure alcohol, the methanol $\mathrm{OH}$ groups bond to small clusters of water at low concentrations $(\sim 50 \mathrm{~mol} . \%) .{ }^{10}$ Moreover, the methanol $\mathrm{CH}_{3}$ groups cluster together in a dense manner and these methyl clusters are connected though the bridging water clusters via $\mathrm{H}$-bonding to the $\mathrm{OH}$ groups.

Naturally, there is a limit to how large these clusters can grow. Above 45 wt.\%, we expect to find water molecules also surrounding the more hydrophobic part of the $n$ PGME molecules and thus act as plasticizer, thereby reduce $T_{g}$ again, as shown in Fig. 1. Also this scenario is supported from diffraction studies of water-methanol. At the concentrations where water-methanol mixtures reach a maximum in viscosity, the structural analysis shows the existence of percolating water clusters. ${ }^{22}$ Thus, at larger water concentrations, water molecules can also surround the hydrophobic parts and thereby the bridging water clusters can grow such that they start to overlap.

In this paper, we have performed a detailed study of the molecular dynamics in the 2PGME-water system at a temperature $(280 \mathrm{~K})$ more than $100 \mathrm{~K}$ above the calorimetric $T_{g}$, using quasielastic neutron scattering (QENS). The results show that the nonmonotonous concentration dependence found at low temperatures ${ }^{17,18}$ is to a very large extent maintained at $280 \mathrm{~K}$. Interestingly, we find that mainly the 2PGME contributes to this slowing down. We also show that the observed dynamics seems to exhibit a peculiar superdiffusive behavior at low momentum transfers $(Q)$.

\section{EXPERIMENTAL}

The QENS measurements were carried out at $280 \mathrm{~K}$ on the high-resolution inverse-geometry back-scattering spectrometer IRIS at the pulsed neutron spallation source International Science Information Service at the Rutherford Appleton Laboratory, United Kingdom. The IRIS spectrometer is described in detail in Ref. 23, so here we will only give some specific details of relevance to the present measurements. Using the PG002 analyzers and an incident neutron wavelength of about $6.6 \AA$, an energy resolution of $17.5 \mu \mathrm{eV}$ [full width at half maximum (FWHM)] and a total energy window of $\pm 0.5 \mathrm{meV}$ were obtained. For all measurements, the samples were confined to an annular volume between two aluminum cylinders. The thickness of the annular samples was chosen to obtain a sample transmission of about 0.9 , which meant a thickness of $0.25 \mathrm{~mm}$ for all samples except those with a high content of $\mathrm{D}_{2} \mathrm{O}$. With these thin samples, we can, to a good approximation, ignore corrections for multiple scattering. The 51 detectors, each corresponding to a specific scattering angle and, therefore, also a specific $Q$-value at zero energy transfer, were grouped into 18 groups of 2-4 detectors per group, giving a total $Q$-range of $0.44-1.85 \AA^{-1}$.

The measured spectra $I_{m}(Q, \omega)$ are convolutions of the scattering law $\mathrm{S}(Q, \omega)$ with the resolution function $\mathrm{R}(Q, \omega)$ of the instrument [in our case determined by a low temperature measurement $(5 \mathrm{~K})$ of the elastic scattering from the 2PGME$\mathrm{H}_{2} \mathrm{O}$ sample containing $30 \mathrm{wt} . \%$ water] in an identical experimental geometry, i.e., $I_{m}(Q, \omega)=S(Q, \omega) \otimes R(Q, \omega)$. By applying a Fourier transform of the measured data, the convolution is reduced to a multiplication in the time domain of the form $I_{m}(Q, t)=I(Q, t) \times R(Q, t)$ (note here the change of notation). The intermediate scattering function $I(Q, t)$ can then easily be obtained by dividing the Fourier transformed data with the Fourier transform of the resolution function $(I(Q, t)$ $\left.=I_{m}(Q, t) / R(Q, t)\right)$. All data treatment as well as the actual numerical Fourier transforms were performed using the onsite program MODES. ${ }^{24}$ This program applies a discrete complex Fourier integral to the measured spectra,

$$
I_{m}(Q, t)=\sum_{k=1}^{N} I_{m}\left(Q, \omega_{k}\right) \exp \left(-i \omega_{k} t\right) \Delta \omega_{k} .
$$

In this equation, $N$ denotes the number of neutron time-offlight channels, $\omega_{k}$ the angular frequency, and $\Delta \omega_{k}$ the width of channel $k$. For our analysis, the data were not subjected to 
binning or interpolation on the energy scale before the numerical Fourier transform was applied in 100 steps (i.e., in 0.01 meV steps) in the energy-transfer range $\Delta E=\mathrm{h} \omega= \pm 0.5$ meV. The drawback of this Fourier transform approach is that it generates small systematic errors, seen as weak oscillations at short times, in $I(Q, t)$ due to the finite number of $\Delta \omega$ steps in the Fourier transform.

In our case, the intermediate scattering function $I(Q, t)$ obtained for each sample and each $Q$-value was adequately described by a single Kohlrausch-William-Watts (KWW) stretched exponential function. ${ }^{25}$ This empirical function is commonly used to describe scattering functions; ${ }^{26}$

$$
I(Q, t)=\exp \left[-\left(\frac{t}{\tau}\right)^{\beta}\right],(0<b<1),
$$

where $\tau$ and $\beta$ are the characteristic relaxation time and the average stretching parameter, respectively. The fact that only one relaxation function was needed to describe the data implies that the different types of molecular motions present in the samples, such as fast rotations of single water molecules and slower translational diffusion of 2PGME molecules, could not be separated in time. Only the average relaxation time, $\langle\tau\rangle$, as a function of $Q$ could be obtained by using this approach to analyze the data. On the other hand, the probably more common approach to fit $\mathrm{S}(Q, \omega)$ with Lorenzian functions, describing exponential relaxations, tend to give somewhat misleading results in cases like this when single exponential relaxations cannot be isolated in the time domain. Furthermore, the relaxation times obtained by this approach tend to depend on the experimental energy resolution. Therefore, we averaged over all dynamics, and the average relaxation time, $\langle\tau\rangle$, was determined from the KWW parameters $\tau$ and $\beta$ by the relation, ${ }^{26}$

$$
\langle\tau\rangle=\frac{\tau}{\beta} \Gamma\left(\frac{1}{\beta}\right),
$$

where $\Gamma$ is the gamma function.

For this study, fully protonated 2PGME, purchased from Sigma-Aldrich and freeze dried to remove small residual impurities, ${ }^{27}$ was mixed with distilled milli-Q $\mathrm{H}_{2} \mathrm{O}$ or $\mathrm{D}_{2} \mathrm{O}$ (from Larodan Fine Chemicals) so that the samples contained $0,15,30,45,60,75$, and 90 wt.\% water (samples with 15 and 45 wt. $\%$ water were not studied in the case of $\mathrm{D}_{2} \mathrm{O}$ ). In the cases when $2 \mathrm{PGME}$ was mixed with $\mathrm{D}_{2} \mathrm{O}$ the rapidly exchangeable $\mathrm{OH}$ end-group was also deuterated by sequential dissolution of $2 \mathrm{PGME}$ in $\mathrm{D}_{2} \mathrm{O}$ followed by distillation. The samples were prepared such that the molar ratio of water to 2PGME was the same for protonated and deuterated samples. A summary of the sample details is shown in Table I. By using both $\mathrm{H}_{2} \mathrm{O}$ and $\mathrm{D}_{2} \mathrm{O}$, it is possible to distinguish between the water dynamics and the motion of 2PGME molecules. The scattering cross-section of $\mathrm{H}$ is at least 1 order of magnitude larger than any other chemical component, including D. This implies that it is mainly the motion of $\mathrm{H}$-atoms that is probed in a material with a reasonably high hydrogen content. Furthermore, the scattering from $\mathrm{H}$ is mainly incoherent, which means that it is basically the self-correlations in space and time that are probed in the experiments. Hence, by replac-
TABLE I. Summary of the sample compositions headings as wt. $\%=$ $m_{\text {water }} / m_{\text {total }}$, Molar ratio $=n_{\text {water }} / n_{2 \text { PGME }}$, Molar fraction $=n_{\text {water/ }} n_{\text {total }}$.

\begin{tabular}{lccc}
\hline \hline Sample & wt. $\% \frac{m_{\text {water }}}{m_{\text {total }}}$ & Molar ratio $\frac{n_{\text {water }}}{n_{2 \text { PGME }}}$ & Molar fraction $\frac{n_{\text {water }}}{n_{\text {total }}}$ \\
\hline 15 wt. $\% \mathrm{H}_{2} \mathrm{O}$ & $15.0 \%$ & 1.4 & $59.2 \%$ \\
30 wt. $\% \mathrm{H}_{2} \mathrm{O}$ & $30.0 \%$ & 3.5 & $77.9 \%$ \\
30 wt. $\% \mathrm{D}_{2} \mathrm{O}$ & $32.1 \%$ & 3.5 & $77.9 \%$ \\
45 wt. $\% \mathrm{H}_{2} \mathrm{O}$ & $44.9 \%$ & 6.7 & $87.0 \%$ \\
60 wt. $\% \mathrm{H}_{2} \mathrm{O}$ & $60.0 \%$ & 12.3 & $92.5 \%$ \\
60 wt. $\% \mathrm{D}_{2} \mathrm{O}$ & $62.3 \%$ & 12.3 & $92.5 \%$ \\
75 wt. $\% \mathrm{H}_{2} \mathrm{O}$ & $75.0 \%$ & 24.6 & $96.1 \%$ \\
75 wt. $\% \mathrm{D}_{2} \mathrm{O}$ & $76.8 \%$ & 24.6 & $96.1 \%$ \\
90 wt. $\% \mathrm{H}_{2} \mathrm{O}$ & $90.0 \%$ & 74.2 & $98.7 \%$ \\
90 wt. $\% \mathrm{D}_{2} \mathrm{O}$ & $90.9 \%$ & 74.4 & $98.7 \%$ \\
\hline \hline
\end{tabular}

ing $\mathrm{H}_{2} \mathrm{O}$ with $\mathrm{D}_{2} \mathrm{O}$, the water dynamics can be almost invisible and only the self-motions of the H-atoms in 2PGME are probed. This is, however, not fully true for the two highest water contents used in this study, e.g., in the case of $2 \mathrm{PGME}$ in 90 wt. $\% \mathrm{D}_{2} \mathrm{O}$ water contributes with almost $30 \%$ to the total scattering in the $Q$-range $<1 \AA^{-1}$. In the $Q$-range around the first peak in the structure factor of $\mathrm{D}_{2} \mathrm{O}$ at about $2.0 \AA^{-1}$, the scattering contribution from $\mathrm{D}_{2} \mathrm{O}$ is as high as $62 \%$ for the sample containing $90 \mathrm{wt} . \% \mathrm{D}_{2} \mathrm{O}$.

By comparing the data for samples prepared with $\mathrm{H}_{2} \mathrm{O}$ and $\mathrm{D}_{2} \mathrm{O}$, respectively, it is possible to subtract the contribution from $2 \mathrm{PGME}$. The $I(Q, t)$ of $2 \mathrm{PGME}$ in $\mathrm{D}_{2} \mathrm{O}$, weighted by the total scattering cross section of the sample at that particular $Q$-value, is subtracted from that of $2 \mathrm{PGME}$ in $\mathrm{H}_{2} \mathrm{O}$ with corresponding weight. Thereafter, the resulting $I(Q, t)$ data are appropriately renormalized so that $I(Q, t)$ becomes unity at $t=0$. With this subtraction procedure, the non-negligible average scattering from $\mathrm{D}_{2} \mathrm{O}$ has been included when the scattering contribution from $2 \mathrm{PGME}$ has been subtracted from the total scattering of the corresponding 2PGME- $\mathrm{H}_{2} \mathrm{O}$ sample. This implies that the contribution from 2PGME is subtracted to a good accuracy but that also a fraction of the scattering from water is subtracted, particularly in the high $Q$-range $\left(Q>1.5 \AA^{-1}\right)$ where the scattering from $\mathrm{D}_{2} \mathrm{O}$ gives a substantial contribution for the two most water rich compositions. However, as long as basically only scattering from water remains, it does not matter if some of the water scattering is removed together with the contribution from 2PGME. The water dynamics can be estimated to a good approximation anyhow.

\section{RESULTS}

Some typical QENS spectra taken at $280 \mathrm{~K}$ and $Q$ $=0.70 \AA^{-1}$ are shown in Fig. 2 together with the low temperature resolution measurement of $2 \mathrm{PGME}-\mathrm{H}_{2} \mathrm{O}$ with $30 \mathrm{wt} . \%$ water. As evident directly from these raw data, the quasielastic broadening is strongly concentration dependent. Moreover, 30 wt.\% water leads to narrower spectral widths compared to both 0 and 90 wt.\%. The concentration dependence of the dynamics can be elucidated in more detail by recourse to the $I(Q, t)$ data presented in Fig. 3. The figure shows $I(Q, t)$ at $Q=0.70 \AA^{-1}$ for $2 \mathrm{PGME}-\mathrm{H}_{2} \mathrm{O}$ and $2 \mathrm{PGME}-\mathrm{D}_{2} \mathrm{O}$ for 


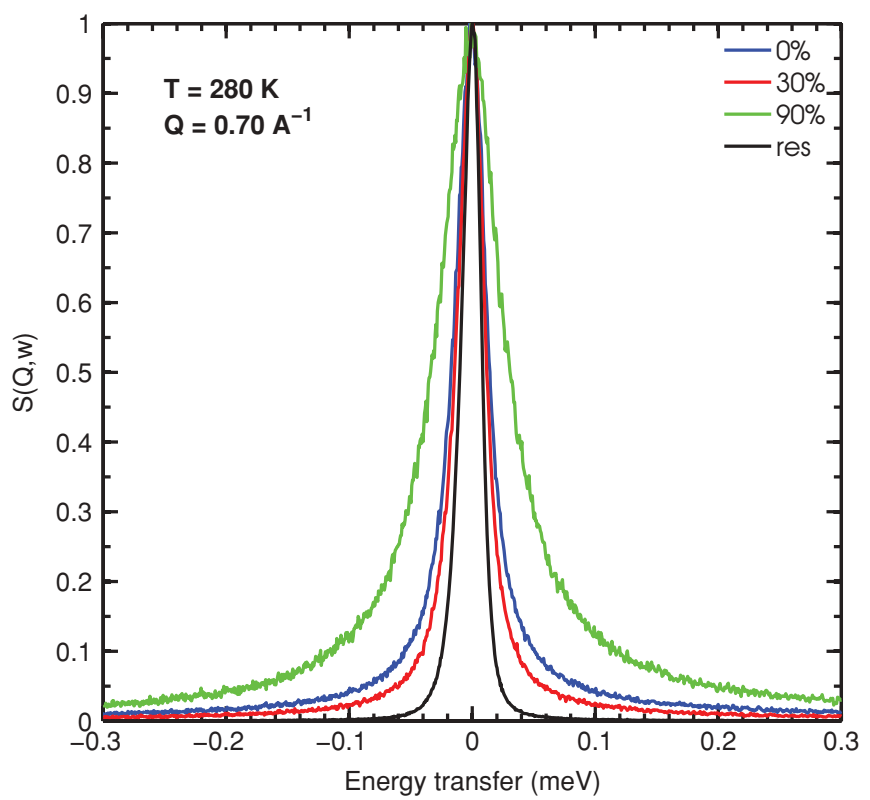

FIG. 2. Representative quasielastic neutron scattering spectra, $S(Q, \omega)$, at $280 \mathrm{~K}$ and $Q=0.70 \AA^{-1}$ for 2 PGME- $\mathrm{H}_{2} \mathrm{O}$ mixtures corresponding to 0 , 30 , and 90 wt. $\%$ water. In addition, a low temperature $(5 \mathrm{~K})$ resolution measurement of $2 \mathrm{PGME}-\mathrm{H}_{2} \mathrm{O}$ with $30 \mathrm{wt} \%$ water is shown to show the instrumental energy resolution $(\mathrm{FWHM}=17.5 \mu \mathrm{eV})$ of the spectrometer. For ease of comparison, all spectra are normalized to unity at zero energy transfer.

different water fractions, as well as for water as obtained by the subtraction procedure described above. Once again, it is clear that the time-scales associated with structural relaxations are strongly dependent on the weight fraction of water in the sample. It is also clear from the data that the relaxation functions are stretched in time and, therefore, best described by the KWW stretched exponential function (Eq. (2) above). The stretching parameter $\beta$ is both sample and $Q$ dependent, and $\beta$ tends to increase with decreasing average relaxation time $\langle\tau\rangle$, which means that it is generally lowest (for a given $Q$-value) for the composition exhibiting the slowest diffusive dynamics and that it increases with increasing $Q$-value. For the lowest $Q$-value of $0.44 \AA^{-1}, \beta$ is in the range $0.5-0.7$ depending on the sample, whereas for the highest $Q$-value of $1.85 \AA^{-1}$, it has increased to a value $>0.7$, approaching unity for the sample with fastest dynamics, i.e., the sample with 90 wt. $\% \mathrm{H}_{2} \mathrm{O}$. In Table II, values of $\tau,\langle\tau\rangle$, and $\beta$ are given for $Q=0.70 \AA^{-1}$.

The average relaxation becomes slowest at about $30 \mathrm{wt} . \%$ water, but there is a difference between the samples with $\mathrm{H}_{2} \mathrm{O}$ and $\mathrm{D}_{2} \mathrm{O}$, respectively, as shown in Fig. 4, which shows the average relaxation time as a function of water concentration. The reason for this difference is, of course, that the fast water dynamics makes a strong contribution to the total scattering in the case of $2 \mathrm{PGME}-\mathrm{H}_{2} \mathrm{O}$ samples and thereby reduces the average relaxation time of the mixture. Since the contribution from water increases with increasing water content, this also leads to a shift of the slowest average relaxation time to a lower water concentration, compared to the $2 \mathrm{PGME}-\mathrm{D}_{2} \mathrm{O}$ system where the scattering from water is substantially lower. From Figs. 3 and 4, it is also evident that the water dynamics speeds up with increasing water content for water concentrations above $30 \mathrm{wt}$. $\%$. In fact, it is possible that the water dynamics speeds up with increasing water content in the whole concentration range from 0 to $100 \mathrm{wt} \%$ water since for very small amounts of water (i.e., less than 1-2 wt.\%) it is likely that the dynamics of these isolated (from other water molecules) water molecules is coupled to the dynamics of the 2PGME molecules, which in pure 2PGME is slightly slower than the water dynamics in the mixture with $30 \mathrm{wt} \%$ water. This speeding up of the water dynamics with increasing water content also contributes to the shift of the slowest average relaxation time to a lower water concentration when 2PGME is dissolved in $\mathrm{H}_{2} \mathrm{O}$ instead of $\mathrm{D}_{2} \mathrm{O}$.

An example of a typical $Q$-dependence of the dynamics is shown in Fig. 5 for $2 \mathrm{PGME}$ with 30 wt. $\% \mathrm{H}_{2} \mathrm{O}$ and $\mathrm{D}_{2} \mathrm{O}$, respectively. $I(Q, t)$ relaxes considerably faster with increasing $Q$, and if we investigate this $Q$-dependence in more detail as shown in Fig. 6, the average relaxation rates in both samples follow a canonical (Fickian) $Q^{2}$ dependence for $Q>1$ $\AA^{-1}$. For the dynamics of the water in the system (i.e., H-D),

TABLE II. Diffusion constants $D$ and values of $\tau,\langle\tau\rangle$, and $\beta$ at $Q=0.70 \AA^{-1}$ are given for the different samples at $T=280 \mathrm{~K}$. Estimated values for the internal water are also provided.

\begin{tabular}{|c|c|c|c|c|}
\hline Sample & $D\left(10^{-10} \mathrm{~m}^{2} / \mathrm{s}\right)$ & $\tau \mathrm{ps}$ & $\langle\tau\rangle \mathrm{ps}$ & $\beta$ \\
\hline 2PGME & $2.9 \pm 0.3$ & $51 \pm 2$ & $74 \pm 5$ & $0.62 \pm 0.02$ \\
\hline 2PGME with 30 wt. $\% \mathrm{D}_{2} \mathrm{O}$ & $1.2 \pm 0.2$ & $157 \pm 4$ & $231 \pm 8$ & $0.59 \pm 0.02$ \\
\hline 2PGME with $60 \mathrm{wt} . \% \mathrm{D}_{2} \mathrm{O}$ & $1.6 \pm 0.2$ & $110 \pm 4$ & $155 \pm 6$ & $0.63 \pm 0.02$ \\
\hline 2PGME with 75 wt. $\% \mathrm{D}_{2} \mathrm{O}$ & $2.6 \pm 0.3$ & $72 \pm 2$ & $93 \pm 4$ & $0.69 \pm 0.02$ \\
\hline 2PGME with 90 wt. $\% \mathrm{D}_{2} \mathrm{O}$ & $4.5 \pm 0.5$ & $40 \pm 2$ & $49 \pm 4$ & $0.73 \pm 0.02$ \\
\hline 2PGME with 15 wt. $\% \mathrm{H}_{2} \mathrm{O}$ & $1.4 \pm 0.2$ & $101 \pm 3$ & $159 \pm 8$ & $0.58 \pm 0.02$ \\
\hline 2PGME with 30 wt. $\% \mathrm{H}_{2} \mathrm{O}$ & $1.5 \pm 0.2$ & $104 \pm 4$ & $151 \pm 7$ & $0.62 \pm 0.02$ \\
\hline 2PGME with 45 wt. $\% \mathrm{H}_{2} \mathrm{O}$ & $2.1 \pm 0.3$ & $83 \pm 3$ & $108 \pm 5$ & $0.68 \pm 0.02$ \\
\hline 2PGME with 60 wt. $\% \mathrm{H}_{2} \mathrm{O}$ & $3.1 \pm 0.4$ & $57 \pm 2$ & $72 \pm 4$ & $0.71 \pm 0.02$ \\
\hline 2PGME with 75 wt. $\% \mathrm{H}_{2} \mathrm{O}$ & $5.2 \pm 0.6$ & $35 \pm 2$ & $41 \pm 3$ & $0.79 \pm 0.02$ \\
\hline 2PGME with $90 \mathrm{wt} . \% \mathrm{H}_{2} \mathrm{O}$ & $9.5 \pm 1.0$ & $21 \pm 1$ & $22 \pm 1$ & $0.88 \pm 0.02$ \\
\hline Water in sample with $30 \mathrm{wt} . \%$ water & $2.3 \pm 0.5$ & $53 \pm 6$ & $67 \pm 10$ & $0.71 \pm 0.03$ \\
\hline Water in sample with $60 \mathrm{wt} . \%$ water & $3.9 \pm 0.7$ & $42 \pm 5$ & $48 \pm 7$ & $0.80 \pm 0.03$ \\
\hline Water in sample with $75 \mathrm{wt} . \%$ water & $5.9 \pm 0.8$ & $31 \pm 3$ & $34 \pm 4$ & $0.84 \pm 0.03$ \\
\hline Water in sample with $90 \mathrm{wt} . \%$ water & $9.8 \pm 1.0$ & $20 \pm 3$ & $21 \pm 3$ & $0.90 \pm 0.03$ \\
\hline
\end{tabular}




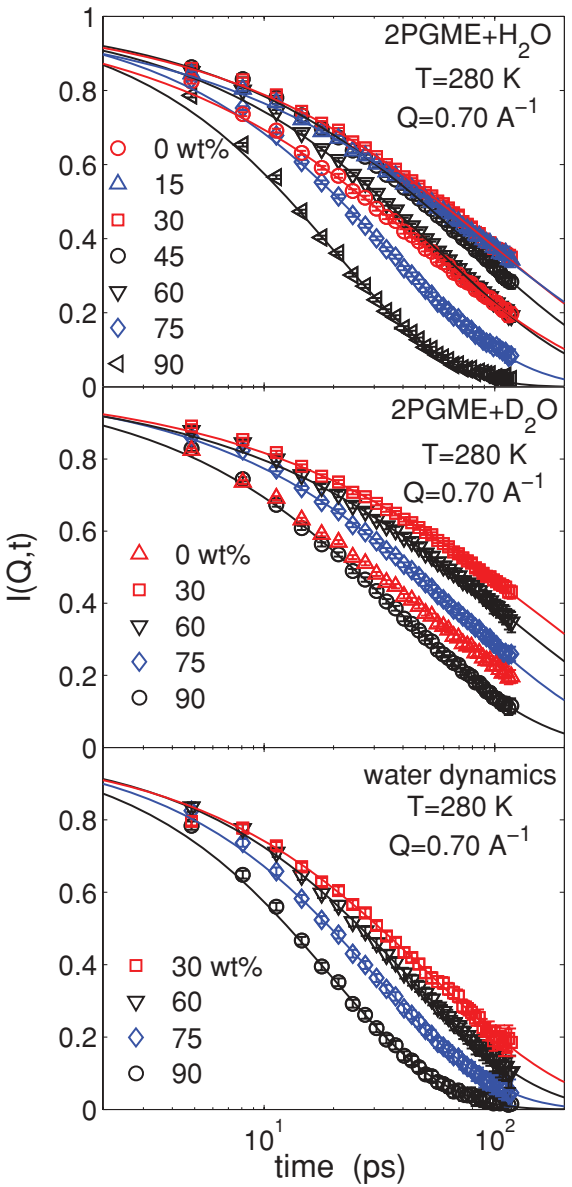

FIG. 3. Intermediate scattering functions, $I(Q, t)$, at $280 \mathrm{~K}$ and $Q=0.70 \AA^{-1}$ for $2 \mathrm{PGME}$-water samples. Water weight fractions are shown in the figures. The upper and middle panels show 2PGME-water samples containing $\mathrm{H}_{2} \mathrm{O}$ and $\mathrm{D}_{2} \mathrm{O}$, respectively, and in the lower panel the water dynamics has been extracted by the subtraction procedure described in Sec. II. The dashed lines are KWW fits [Eq. (2)] to the experimental data points.

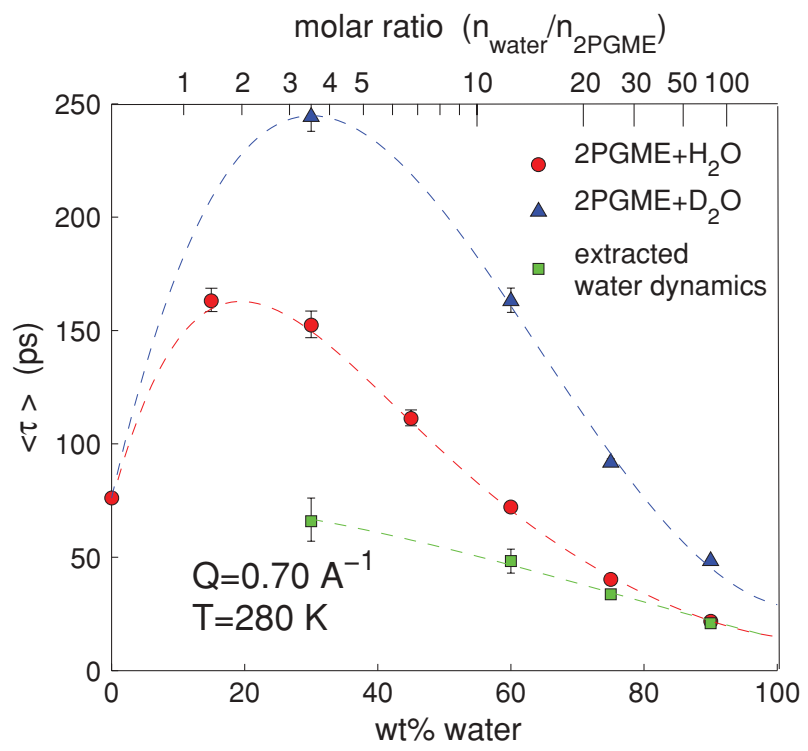

FIG. 4. Average relaxation times, $\langle\tau\rangle$, determined by Eq. (3) from the $\tau$ and $\beta$ parameters obtained from the KWW fits shown in Fig. 3. $\langle\tau\rangle$ as a function of the wt.\% water is shown for 2PGME-water samples of both $\mathrm{H}_{2} \mathrm{O}$ and $\mathrm{D}_{2} \mathrm{O}$. Characteristic relaxation times related to the water dynamics have been obtained via recourse to the subtraction procedures described in the text. The dashed lines are polynomial fits to the data points.

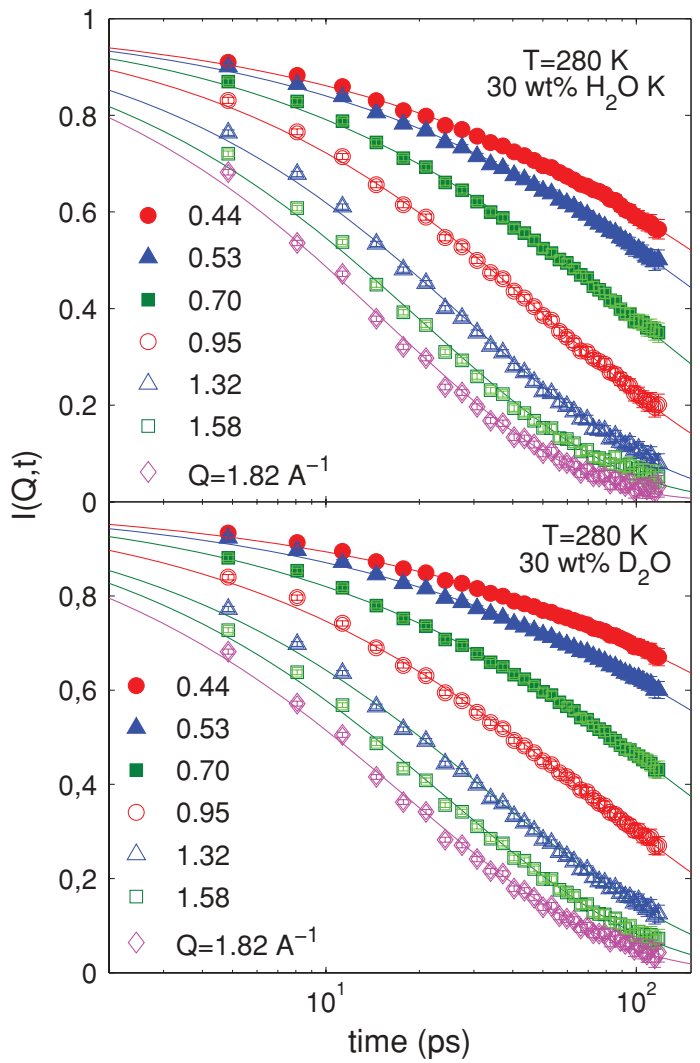

FIG. 5. Intermediate scattering functions, $I(Q, t)$, at $280 \mathrm{~K}$ for $2 \mathrm{PGME}-\mathrm{H}_{2} \mathrm{O}$ (upper panel) and $2 \mathrm{PGME}-\mathrm{D}_{2} \mathrm{O}$ (lower panel) with $30 \mathrm{wt} \% \%$ water. Data are shown for the $Q$-values indicated in the figures and the dashed lines are KWW fits [Eq. (2)] to the experimental data points.

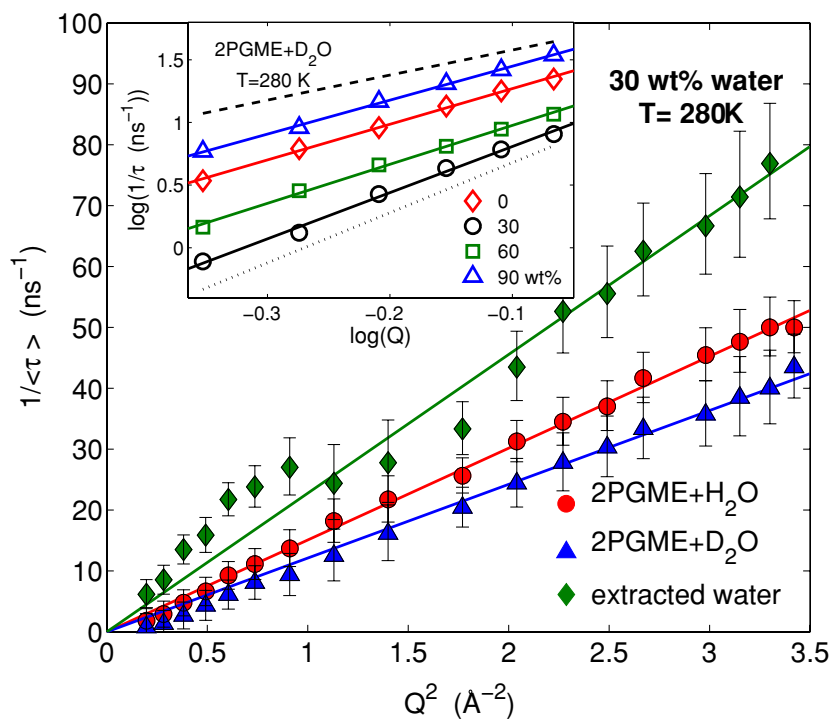

FIG. 6. Reciprocal average relaxation times, $1 /\langle\tau\rangle$, determined by Eq. (3) from the $\tau$ and $\beta$ parameters obtained from the KWW fits shown in Fig. 5. $1 /\langle\tau\rangle$ is shown as a function of $Q^{2}$, which should give a linear dependence for continuous translational diffusion. The lines indicate the average diffusion constants, $D$, (presented in Table II) of $2 \mathrm{PGME}-\mathrm{H}_{2} \mathrm{O}$ and $2 \mathrm{PGME}-\mathrm{D}_{2} \mathrm{O}$ with $30 \mathrm{wt} . \%$ water, as well as the extracted water contribution, at $T=280 \mathrm{~K}$. The inset shows logarithmic (base 10) representations of $1 /\langle\tau\rangle$ as a function of $Q$ for 2PGME- $\mathrm{D}_{2} \mathrm{O}$ samples with $0,30,60$, and $90 \mathrm{wt} . \%$ water. In this low $Q$-region, the $Q$-dependence of $1 /\langle\tau\rangle$ is fitted to $Q^{a}$ (solid lines), where the exponent $a \approx 2.7$ for most of the water concentrations. The dashed and dotted lines represent the $Q^{2}$ - and $Q^{4}$-dependencies, respectively. 
this $Q^{2}$ dependence seems to be valid over the entire $Q$-range, although the subtraction procedure used to extract the water dynamics increases the error bars. A $Q^{2}$ dependence is a characteristic feature of stochastic translational diffusion, which, therefore, is the dominating dynamics. As shown in Fig. 6, diffusion constants, $D$, have been estimated from linear fits to all data points according to the relation $D=1 /\langle\tau\rangle Q^{2} .^{28,29}$ The so obtained values are given in Table II for all samples investigated.

For $Q<1 \AA^{-1}$ we see in Fig. 6 that the inverse relaxation time exhibits an even stronger $Q$-dependence, particularly in the case of $2 \mathrm{PGME}$ in $\mathrm{D}_{2} \mathrm{O}$. In the inset of Fig. 6 this $Q$-dependence is shown in a $\log \left(\langle\tau\rangle^{-1}\right)$ versus $\log (Q)$ plot for 2PGME at different $\mathrm{D}_{2} \mathrm{O}$ concentrations. For most of the water concentrations, $\langle\tau\rangle^{-1}$ is proportional to approximately $Q^{2.7}$ (although the error bars are large due to the limited experimental time range of the spectrometer). This finding suggests that the relaxation of the 2PGME molecules is somewhat intrinsically stretched, as is further discussed below.

\section{DISCUSSION}

The observed maximum in the viscosity related dynamics at an intermediate composition of the 2PGME-water system cannot be considered to be normal behavior, although it is neither unique for highly nonideal solutions. As mentioned above, alcohol-water mixtures and some other aqueous solutions show a similar concentration dependence. However, the present findings for the 2PGME-water system are nevertheless rather unique since the same explanations as previously proposed cannot be used to explain the anomalous concentration dependence of the dynamics in this system. In contrast to other investigated aqueous systems, where the water dynamics has mainly been responsible for viscosity maxima, this study indicates that it is mainly the slowing down of the motion of 2PGME molecules with increasing water concentration up to the concentration in the vicinity of the viscosity maximum that is responsible for the observed behavior. At $30 \mathrm{wt} . \%$ water, the characteristic relaxation time of $2 \mathrm{PGME}$ is three times longer than that of pure 2PGME. This slowing down occurs even though the water dynamics (at $30 \mathrm{wt} . \%$ ) is faster than that of pure 2PGME.

A structural model similar to the one presented in Introduction of this paper can be used to explain the anomalous concentration dependence of the dynamics in the 2PGMEwater system. Previous studies ${ }^{19,20}$ show that even in a pure liquid of 2PGME, the molecular dynamics is slower than expected for a single 2PGME molecule due to the formation of $\mathrm{H}$-bonded molecular pairs. It is then expected that added water molecules have a larger tendency to coordinate to the $\mathrm{OH}$ end-groups than the 2PGME molecules and thereby forming "bridges" between 2PGME molecules. This leads to an increasing size, and thereby also slower motions, of the effective relaxing units with increasing water concentration up to a saturation point at a certain water content. Also the fact that the water-2PGME interactions are stronger than the 2PGME2PGME interactions should contribute to the slowing down of the dynamics in this concentration range. However, there seems to be faster motions of water within the clusters. As the water clusters grow, these internal motions speed up since, on average, less coupling to 2PGME occurs. Thus, the dynamics of water and 2 PGME can have different concentration dependencies. A saturation point is reached due to the fact that the water bridges cannot be infinitely large due to the short lifetime of the hydrogen bonds. Above the saturation point, the additional water will instead be more free and mobile and have a plasticizing effect on the 2PGME molecules, leading to an increasing mobility of them with increasing water content. ${ }^{18}$

The $T_{g}$ maximum at a water content of about 45 wt. $\%$ implies that there is a viscosity maximum at the same water concentration in the temperature range around the glass transition, i.e., in the range 170-185 K. Thus, the calorimetric measurements presented in Ref. 18 suggest that the slowest dynamics should be obtained at a water content of about 45 wt. $\%$, provided that the concentration dependence of the dynamics is temperature independent. However, in this QENS study performed at $T=280 \mathrm{~K}$ the slowest mobility of the 2PGME molecules was obtained at about $30 \mathrm{wt} \%$ water, rather than at 45 wt.\% water, indicating that the saturation point is temperature dependent. This finding can be explained by the fact that the local motions of the water molecules are slower at low temperatures close to $T_{g}$ than at $280 \mathrm{~K}$. The size of the bridging water clusters are then expected to decrease with increasing temperature, in agreement with the experimental results. Also this picture is supported by diffraction studies. The nanosegregation found in water-methanol mixtures is enhanced as the temperature is decreased. ${ }^{30}$ Thus, the maximum size of the effective structures at deeply supercooled temperatures should exceed those at room temperature and likewise, the concentration which exhibits the slowest dynamics should be higher at $T_{g} \cdot{ }^{30}$

In the case of the present 2PGME-water system, where we know that no polymerlike structures are developed, the observed $Q$-dependence can be discussed in terms of heterogeneous and homogenous dynamical scenarios. ${ }^{31,32}$ In the heterogeneous case, the stretching of $I(Q, t)$ is due to a superposition of different single exponential relaxations from relaxing units with different relaxation times. In the homogeneous case, all units relax identically but nonexponentially. Thus, in this case each relaxation process is characterized by the same stretched exponential. In the case of homogeneous dynamics, $\beta$ is considered as an indicator of the degree of correlation or cooperativity of the relaxation process. Thus, both the homogeneous and the heterogeneous scenarios may give rise to the same stretched $\mathrm{KWW}$ function of $I(Q, t)$ but the two scenarios can be distinguished by their different $Q$-dependences of $\langle\tau\rangle$. In the heterogeneous case, it has been shown that $\langle\tau\rangle \propto 1 / Q^{2}$ and in the homogeneous case $\langle\tau\rangle \propto$ $1 / Q^{2 / \beta}{ }^{31}$ Thus, a true homogeneous scenario should give an exponent $n$ in the range $3-4$ in the $Q$-range $<1 \AA^{-1}$ where the $\beta$ values for the $2 \mathrm{PGME}-\mathrm{D}_{2} \mathrm{O}$ samples are in the range $0.50-0.75$. The $n$ value of approximately 2.7 in this study indicates that we have an intermediate case between the heterogeneous and homogeneous scenarios. It should here be noted that similar $Q$-dependences have previously been 
observed for other molecular liquids, such as glycerol, ${ }^{33}$ 1-n-butyl-3-methylimidazolium hexafluorophosphate, ${ }^{34}$ and ethylene glycol. ${ }^{35}$ Our finding suggests that the diffusive relaxation dynamics of the 2PGME molecules is somewhat intrinsically stretched but the stretched relaxation function is also due to that it involves a number of different relaxation processes occurring on different time scales.

In the high $Q$-range ( $>1 \AA^{-1}$ ), we find to a good approximation that $\langle\tau\rangle \propto 1 / Q^{2}$ (at least up to the maximum $Q$-value of $1.85 \AA^{-1}$ ). This weaker $Q$-dependence in the high $Q$-range is related to the observation that the stretching parameter $\beta$ increases with increasing $Q$-value. For $Q>1 \AA^{-1}, \beta$ becomes rather close to unity and both the heterogeneous and the homogeneous scenarios approach the same $\langle\tau\rangle \propto 1 / Q^{2}$ dependence, in agreement with the experimental findings. Thus, the increase of $\beta$ with increasing $Q$ together with the partly homogeneous stretching of $I(Q, t)$ accounts for the observation of a $\langle\tau\rangle \propto 1 / Q^{2.7}$ dependence in the low $Q$-range and a more common Fickian $\langle\tau\rangle \propto 1 / Q^{2}$ dependence at high $Q$.

\section{CONCLUSIONS}

The present QENS study on 2PGME-water mixtures of different water contents shows that the relaxation dynamics and diffusivity of the 2PGME molecules exhibit nonideal dependences on water concentration. Instead of a monotonic concentration dependence, as expected from an ideal solution, the dynamics slows down with increasing water content for water concentrations up to about $30 \mathrm{wt}$. \%. In previous studies of other aqueous mixtures where such nonmonotonic behavior is found, it is often argued that it is the water molecules that primarily slow down with water content at low concentrations. However, this study indicates that the dynamics of the water molecules exhibits a rather monotonic concentration dependence and that it is the 2PGME that significantly slows down and reaches a diffusivity minimum at $30 \mathrm{wt} . \%$ water. The finding is in qualitative agreement with the observation of a maximum in $T_{g}$ at about 45 wt.\% water (see Fig. 1 and Ref. 18), although it should be noted that the present results are taken at $280 \mathrm{~K}$, i.e., more than $100 \mathrm{~K}$ above the $T_{g}$ maximum. It is difficult to explain such a slowing down of the 2PGME dynamics with increasing water content without introducing some kind of structural alteration. The present results indicate that bridging of water cluster between 2PGME molecules can account for the slowing down as larger effective dynamic units are created. The increasing size of these relaxing units can then explain the decreasing mobility of the 2PGME molecules with increasing water content. However, the clusters of bridging water molecules cannot be infinitely large due to the short lifetime of the hydrogen bonds. Instead, a saturation point will be reached at a certain water content, where additional water is more free and mobile and have a plasticizing effect on the 2PGME molecules, leading to an increasing mobility with increasing water content.

\section{ACKNOWLEDGMENTS}

The authors are grateful to Dr. M. Furlani for valuable help during the sample preparation. This work was financially supported by the Swedish Research Council and the Swedish Energy Agency.

${ }^{1}$ S. J. Suresh and V. M. Naik, J. Chem. Phys. 113, 9727 (2000).

${ }^{2}$ Biophysics of Water, edited by F. Franks and S. Mathias (Wiley, London, 1983).

${ }^{3}$ M. Ageno and C. Frontali, PNAS 57, 856 (1967).

${ }^{4}$ S. Akhtar M. M. H. Bhuiyan, M. S. Uddin, B. Sultana, M. Nessa, and M. A. Saleh, Phys. Chem. Liq. 37, 215 (1999).

${ }^{5}$ K. A. Valiev and M. I. Emel'yanov, Zhurnal Strukturnoi Khimii 5, 7 (1964).

${ }^{6}$ H. S. Frank and M. W. Evans, J. Chem. Phys. 13, 507 (1945).

${ }^{7}$ R. F. Lana and B. C.-Y. Lu, J. Chem. Eng. Data 10, 216 (1965).

${ }^{8}$ A. K. Soper and J. L. Finney, Phys. Rev. Lett. 71, 4346 (1993).

${ }^{9}$ D. T. Bowron, A. K. Soper, and J. L. Finney, J. Chem. Phys. 114, 6203 (2001).

${ }^{10}$ S. Dixit, J. Crain, W. C. K. Poon, J. L. Finney, and A. K. Soper, Nature (London) 416, 829 (2002).

${ }^{11}$ M. Misawa, Y. Inamura, D. Hosaka, and O. Yamamuro, J. Chem. Phys. 125, 074502 (2006).

${ }^{12}$ M. Misawa, K. Yoshida, K. Maruyama, H. Munemura, and Y. Hosokawa, J. Phys. Chem. Solids 60, 1301 (1999).

${ }^{13}$ V. Calandrini, A. Deriu, G. Onori, R. E. Lechner, and J. Pieper, J. Chem. Phys. 120, 4759 (2004).

${ }^{14}$ M. Nakada, K. Maruyama, O. Yamamuro, and M. Misawa, J. Chem. Phys. 130, 074503 (2009).

${ }^{15}$ G. C. Benson and O. Kiyohara, J. Solution Chem. 9, 791 (1980).

${ }^{16}$ H. N. Bordallo, K. W. Herwig, B. M. Luther, and N. E. Levinger, J. Chem. Phys. 121, 12457 (2004).

${ }^{17}$ J. Sjöström, J. Mattsson, R. Bergman, E. Johansson, K. Josefsson, D. Svantesson, and J. Swenson, Phys. Chem. Chem. Phys. 12, 10452 (2010).

${ }^{18}$ J. Sjöström, J. Mattsson, R. Bergman, and J. Swenson (in manuscript).

${ }^{19}$ J. Mattsson, R. Bergman, P. Jacobsson, and L. Börjesson, Phys. Rev. B 79, 174205 (2009).

${ }^{20}$ A. Bernson and J. Lindgren, Polymer 35, 4842 (1994).

${ }^{21}$ A. K. Soper, Mol. Phys. 99, 1503 (2001).

${ }^{22}$ L. Dougan, S. P. Bates, R. Hargreaves, J. P. Fox, J. Crain, J. L. Finney, V. Réat, and A. K. Soper, J. Chem. Phys. 121, 6456 (2004).

${ }^{23}$ C. J. Carlile and M. A. Adams, Physica B 182, 431 (1992).

${ }^{24}$ W. S. Howells, V. Garcia Sakai, F. Demmel, M. T. F. Telling, and F. Fernandez-Alonso, The MODES User Guide version 3, Rutherford Appleton Laboratory Technical Reports RAL-TR-2010-006 (2010).

${ }^{25}$ G. Williams and D. Watts, Trans. Faraday Soc. 66, 80 (1970).

${ }^{26}$ G. W. Sherer, J. Non-cryst. Solids 123, 75 (1990).

${ }^{27}$ J. Sjöström, R. Bergman, J. Swenson, C. Wadell, T. Moberg, and J. Mattsson, "The effect of water contamination on the supercooled dynamics of a hydrogen bonded model glass former," submitted to J. Phys. Chem. B.

${ }^{28}$ M. Bée, Quasielastic Neutron Scattering: Principles and Applications in Solid State Chemistry, Biology and Material Science (Adam Hilger, Bristol and Philadelphia, 1988).

${ }^{29}$ S. Longeville, W. Doster, M. Diehl, R. Gähler, and W. Petry, in Neutron Spin Echo Spectroscopy, edited by F. Mezei, C. Cappas, and T. Gutberlet (Springer-Verlag, Berlin, 2003), p. 325.

${ }^{30}$ L. Dougan, R. Hargreaves, S. P. Bates, J. L. Finney, V. Réat, A. K. Soper, and J. Crain, J. Chem. Phys. 122, 174514 (2005).

${ }^{31}$ A. Arbe, J. Colmenero, M. Monkenbusch, and D. Richter, Phys. Rev. Lett. 81, 590 (1998).

${ }^{32}$ J. Colmenero, A. Arbe, A. Alegia, M. Monkenbusch, and D. Richter, J. Phys.: Condens. Matter 11, A363 (1999).

${ }^{33}$ J. Wuttke, W. Petry, G. Goddens, and F. Fujara, Phys. Rev. E 52, 4026 (1995)

${ }^{34}$ A. Triolo, O. Russina, V. Arrighi, F. Juranyi, S. Janssen, and C. M. Gordon, J. Chem. Phys. 19, 8549 (2003).

${ }^{35}$ O. Sobolev, A. Novikov, and J. Pieper, Chem. Phys. 334, 36 (2007). 